\title{
Comprehensive analysis of coding-IncRNA gene co-expression network uncovers conserved functional IncRNAs in zebrafish
}

Wen Chen ${ }^{1,2+}$, Xuan Zhang ${ }^{1 \dagger}$, Jing $\mathrm{Li}^{1+}$, Shulan Huang ${ }^{2}$, Shuanglin Xiang ${ }^{2}$, Xiang $\mathrm{Hu}^{2^{*}}$ and Changning $\mathrm{Liu}^{1 *}$

From The Sixteenth Asia Pacific Bioinformatics Conference 2018

Yokohama, Japan. 15-17 January 2018

\begin{abstract}
Background: Zebrafish is a full-developed model system for studying development processes and human disease. Recent studies of deep sequencing had discovered a large number of long non-coding RNAs (IncRNAs) in zebrafish. However, only few of them had been functionally characterized. Therefore, how to take advantage of the mature zebrafish system to deeply investigate the IncRNAs' function and conservation is really intriguing.

Results: We systematically collected and analyzed a series of zebrafish RNA-seq data, then combined them with resources from known database and literatures. As a result, we obtained by far the most complete dataset of zebrafish IncRNAs, containing 13,604 IncRNA genes (21,128 transcripts) in total. Based on that, a co-expression network upon zebrafish coding and IncRNA genes was constructed and analyzed, and used to predict the Gene Ontology (GO) and the KEGG annotation of IncRNA. Meanwhile, we made a conservation analysis on zebrafish IncRNA, identifying 1828 conserved zebrafish IncRNA genes (1890 transcripts) that have their putative mammalian orthologs. We also found that zebrafish IncRNAs play important roles in regulation of the development and function of nervous system; these conserved IncRNAs present a significant sequential and functional conservation, with their mammalian counterparts.

Conclusions: By integrative data analysis and construction of coding-IncRNA gene co-expression network, we gained the most comprehensive dataset of zebrafish IncRNAs up to present, as well as their systematic annotations and comprehensive analyses on function and conservation. Our study provides a reliable zebrafish-based platform to deeply explore IncRNA function and mechanism, as well as the IncRNA commonality between zebrafish and human.
\end{abstract}

Keywords: LncRNA, Zebrafish, Co-expression network, Gene ontology, KEGG, Conservation

\section{Background}

LncRNAs are termed as a heterogeneous class of transcripts with the length over $200 \mathrm{bp}$ and without the potential of protein-coding [1-3]. By now, there are ten thousands of lncRNAs discovered across human, mouse, nematode, zebrafish etc. [4-6]. Meanwhile, an increasing number of studies demonstrated that IncRNAs have

\footnotetext{
* Correspondence: huxiang@hunnu.edu.cn; liuchangning@xtbg.ac.cn ${ }^{\dagger}$ Equal contributors

${ }^{2}$ State Key Laboratory of Developmental Biology of Freshwater Fish, School of Life Sciences, Hunan Normal University, Changsha, China

${ }^{1}$ Key Laboratory of Tropical Plant Resources and Sustainable Use,

Xishuangbanna Tropical Botanical Garden, Chinese Academy of Sciences, Kunming, China
}

special functions in diverse biological processes and can network for gene expression, mutation and dysregulation of them are likely linked to diverse human diseases and abnormal development [10-12].

Zebrafish as a powerful model for studying vertebrate biology has a relatively long history, on account of its clear development pattern and genetic background [13]. Large-scale genetic screens have identified hundreds of mutant phenotypes, many of which could imitate human status, and thus influencing cellular homostasis [7-9]. As a result, these enormous amounts of lncRNAs can 
disease status, providing a simple and accessible system for investigating the corresponding human diseases [14-16]. At the early stage of this kind of research, the focal spot is primarily on protein-coding gene. The well-characterized cases of using zebrafish as a model for studying protein roles in human disease are coming from the hematopoietic diseases, like ALAS2 in a microcytic, hypochromic anemia, UROD in porphyria, and etc. [17]. The semblable studies are also illuminated in Cardiovascular-, Kidney- and other organ- disorders $[18,19]$. However, the similar studies of using the zebrafish as a model to probe lncRNAs' function are still at infancy, because of the insufficient annotation and the lack of systematic survey.

To date, coupled with the progress of next generation sequencing in model organisms, it is feasible of large-scale discovering and annotating and integratively computational analyzing of zebrafish lncRNAs. By using chromatin marks, poly(A)-site mapping and RNA-Seq data, Ulitsky et.al had identified 550 distinct lncRNAs in zebrafish, with 29 lncRNAs having detectable sequence similarity with their putative mammalian orthologs and some having conserved genomic locations [20]. By a time-series of RNA-seq experiments, Andrea Pauli et al. had defined 1133 noncoding multi-exonic transcripts expressed at eight stages during early zebrafish embryogenesis, and found that zebrafish lncRNAs share many of the characteristics of their mammalian counterparts [21].

In this work, to better utilize the mature zebrafish system to uncover the commonality of lncRNAs' function and conservation between zebrafish and human, and further provide a research platform of zebrafish model organisms to explore the function of lncRNAs in development processes and human disease, we presented and analyzed the most complete collection and annotation of zebrafish lncRNAs, by comprehensively integrating various data resources from public RNA-seq datasets, databases and literatures. By establishing a coexpression network upon lncRNA- and coding- genes, we set out to optimize the present gene annotation of zebrafish lncRNAs, to predict their potential functions, and to hunt their putative mammalian orthologs, so as to illuminate their functional and mechanism conservation between species.

\section{Results}

Comprehensive collection and integrative data analysis of zebrafish IncRNAs

To acquire the zebrafish lncRNAs as comprehensive as possible, we made a pipeline for data collection, integration and annotation (Fig. 1a). First, we collected all possible lncRNAs of zebrafish from publicly available databases and literatures, and further integrated them with RNA-seq datasets covering 499 runs in 56 studies from NCBI SRA database (Additional file 1). Using the in-house "RNA-seq data analysis pipeline", we obtained our final dataset with more zebrafish lncRNAs, containing in total 13,604 lncRNA genes (21,128 transcripts) (Additional file 2). The major data sources of our lncRNA set are from RNA-seq data analysis and NONCODE [22], followed by NCBI, Ensembl and zflncRNApedia orderly [23-25] (Fig. 1b). Venn diagram shows that, although there is an obvious overlapping between different sources, a lot of unique lncRNAs emerged in RNA-seq datasets and NONCODE (Fig. 1c).

According to the genomic location, these zebrafish lncRNA genes are cataloged as intergenic, sense overlapping, antisense and intronic, in the percentage of $43.9 \%$, $27.0 \%, 26.8 \%$ and $2.3 \%$ respectively (Fig. 2a). Similar to coding-gene, zebrafish lncRNA genes have a relatively uniform distribution on chromosome, but less density. There are about 100 lncRNA genes per $10 \mathrm{M}$ sequence, while coding-genes are about 200 genes per $10 \mathrm{M}$ sequence (Fig. 2b). As for the number of isoform for each gene, lncRNA genes are also in line with codinggenes: (1) about $76.1 \%$ of lncRNA genes are single-isoform genes, which occupy $66.5 \%$ in coding-genes; (2) lncRNA genes with isoform $\leq 3$ account to $94.2 \%$, corresponding to $94.1 \%$ of coding-genes; (3) lncRNA genes with isoform $\geq 4$ only have $5.8 \%$ percentage, consistent with $5.9 \%$ of codinggenes (Fig. 2c). However, the exon number distribution of zebrafish lncRNAs is far from that of coding-gene, in that the amount of transcripts with multi-exons is significantly less (Fig. 2d). About 40\% of zebrafish lncRNA transcripts have two exons, but for coding-genes, the percentage is only $6.7 \%$; in reverse, transcripts with exon number $\geq 5$ are increasing in coding-genes (75\%), compared with lncRNAs (17.5\%).

\section{Construction of zebrafish coding-IncRNA gene co-expression network}

To establish the coding-lncRNA gene co-expression network of zebrafish, we used all collected RNA-seq datasets to quantify the expression levels of both coding- and lncRNAgenes in different tissues and conditions (Additional file 3). The genes with high-expressional variance (top 75 percentile) were selected for identification of co-expressed gene-pairs using Spearman's correlation coefficient. The $P$-value was estimated by Fisher transformation, and the sets of $P$-value for each gene were adjusted by the Bonferroni method. Only gene-pairs with an adjusted $P$-value of 0.01 or less are retained in subsequent analyses.

By comparison of the correlation coefficients of gene expression between coding-genes, between lncRNA and coding-gene, and between lncRNAs, respectively (Fig. 3a), we found that the expression correlation between interlncRNAs is obviously weaker than inter-coding-genes (Kolmogorov-Smirnov test, $P$-value $=1.45 \mathrm{e}-232$ ). Intriguingly, the expression correlation between lncRNA and 

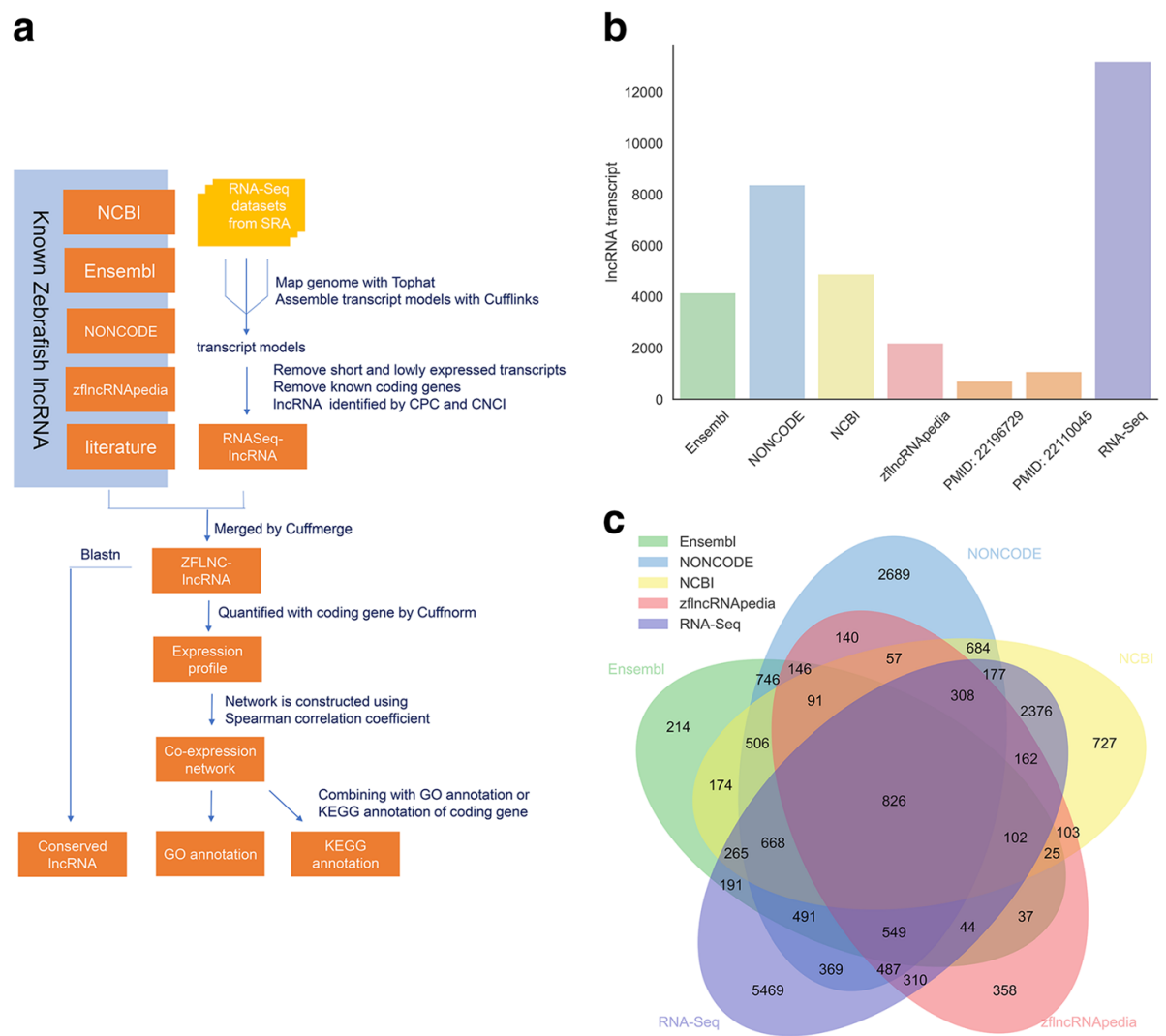

Fig. 1 Integration of all sources of zebrafish IncRNA. a Data sources and analysis pipeline. $\mathbf{b}$ The number of IncRNA transcripts from each source. c Venn diagram between different sources

coding-gene is visibly enhanced, as compared with interlncRNAs (Kolmogorov-Smirnov test, $P$-value $=3.49 \mathrm{e}-33$ ), which is likely due to the functional synergia of lncRNAs with their protein partners.

We further evaluated the networks using different cutoffs of Spearman's correlation coefficient by several network parameters (Fig. 3b). With the ascending of cutoff value, the network size, that is consisting of both coding- and lncRNA- genes, naturally decreased. Yet, the average clustering coefficient and the transitivity scores, two parameters that reflect small-world characters of gene network, still held steady or slightly declined. Moreover, coding-genes having 10 or more known coding neighbors were used as a test set to evaluate the functional prediction performance for the network with different cutoffs (Fig. 3c). We found that, for each cutoff, its prediction performance is higher than randomly selected node; furthermore, the prediction sensitivity increases as the cutoff rises, since more GO annotations of coding-genes can be precisely predicted by their neighbor nodes. This indicated that the higher the cutoff, the more similar the annotated functions of connected gene-pairs in network.

According to the size and quality of these networks, we selected the network that has a Spearman's correlation coefficient cutoff of 0.5 for follow-up analysis. This coding-lncRNA gene co-expression network shows a scale-free distribution of connectivities (Fig. 3d). There were 9015 non-coding-genes and 24,425 coding-genes that were linked by $25,097,918$ edges. Nearly $17,800,940$ edges (70.93\%) were connected among coding-genes, and $6,253,398$ edges (24.92\%) were connected among codingand noncoding- genes, whereas another 1,043,580 edges (4.16\%) were linked between pairs of noncoding- genes.

\section{Functional annotation of zebrafish IncRNAs according to coding-IncRNA gene co-expression network}

In our co-expression network established between codingand lncRNA- gene, the connected gene-pairs tend to have more similar annotated functions, thereby we could use it to further annotate zebrafish IncRNAs' function, including GO and KEGG pathway. Of the 24,425 coding-genes in this network, 14,650 (59.98\%) and 7022 (28.75\%) were annotated with at least one GO Biological Process (BP) or KEGG term. Consequently, using functional enrichment analysis by hypergeometric distribution test ( $p$-value $<0.05)$, we annotated each zebrafish lncRNA according to its immediate neighbor coding-genes that have known functional annotation. In this way, we achieved a set of GO (7345 genes) and 


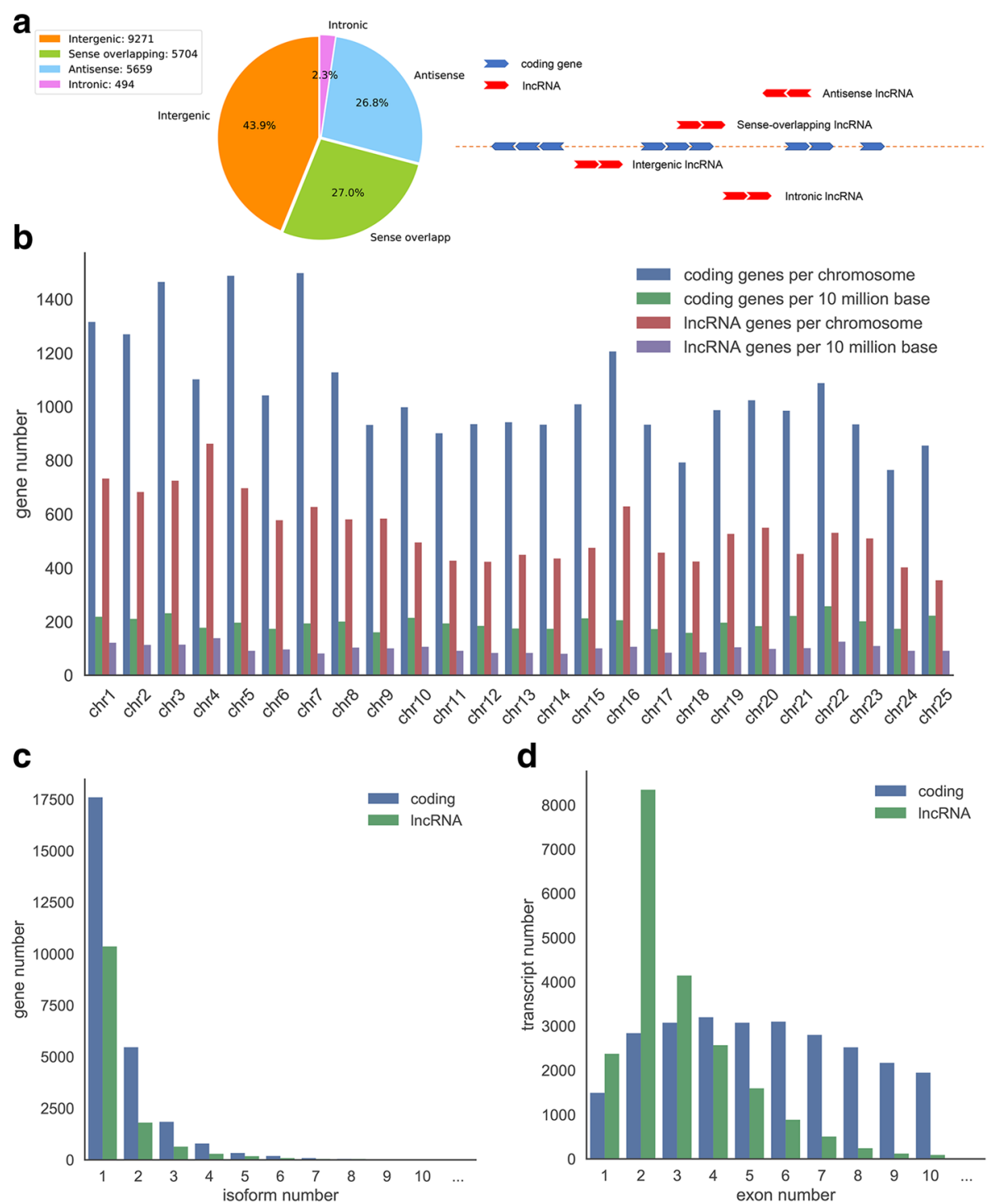

Fig. 2 Features of Zebrafish IncRNA. a Distribution of IncRNA subtypes. b Zebrafish IncRNA distribution in chromosomes. c Distribution of zebrafish IncRNA isoform number. $\mathbf{d}$ Distribution of zebrafish IncRNA exon number

KEGG (7055 genes) annotation of zebrafish lncRNAs, as listed in Additional file 4.

In terms of $\mathrm{GO}$ annotation, the top two enriched GO BP terms of zebrafish lncRNAs are associated with chromosome-related processes, as 'transposition' $(P$-value $=1.33 \mathrm{e}-11)$ and 'chromosome segregation' $(P$-value $=1.32 \mathrm{e}-10) \quad($ Fig. $4 \mathrm{a})$. This finding is consistent with previous researches of mammalian lncRNAs, suggesting that lncRNAs can act in the nucleus to regulate the activity of chromosome. For instance, XIST lays over the $\mathrm{X}$ chromosome in cis and balances $\mathrm{X}$-linked gene expression [26]; AIR regulates genomic imprinting of a cluster of autosomal genes in cis [27]; else, NORAD controls chromosome segregation and protects cell from becoming aneuploid [28].
In addition, compared with coding-genes, we found that the top ten GO BP terms of zebrafish lncRNAs more tend to be enriched in cell-signaling-related or nervous-system-related processes, such as 'cell-cell signaling' $(P$-value $=4.33 \mathrm{e}-10)$, 'cell junction organization' $(P$-value $=2.1 \mathrm{e}-10),{ }^{\prime}$ 'homeostatic process' $(P$-value $=3.56 \mathrm{e}-10)$ and 'neurological system process' $(P$-value $=4.5 \mathrm{e}-10)$. Interestingly, this finding is intensely echoed by the results of KEGG annotation, in that the top ten KEGG pathways of zebrafish lncRNAs are subtotally correlated to cell signaling and nervous system (Fig. 4b), such as 'Glutamatergic synapse $(P$-value $=5.9 \mathrm{e}-320)$, 'Morphine addiction' $(P$-value $=$ $5.9 \mathrm{e}-320)$, 'Neuroactive ligand-receptor interaction' $(P$-value $=5.02 \mathrm{e}-186)$, and 'Retrograde endocannabinoid signaling' $(P$-value $=6.2 \mathrm{e}-182)$. Since previous studies have 

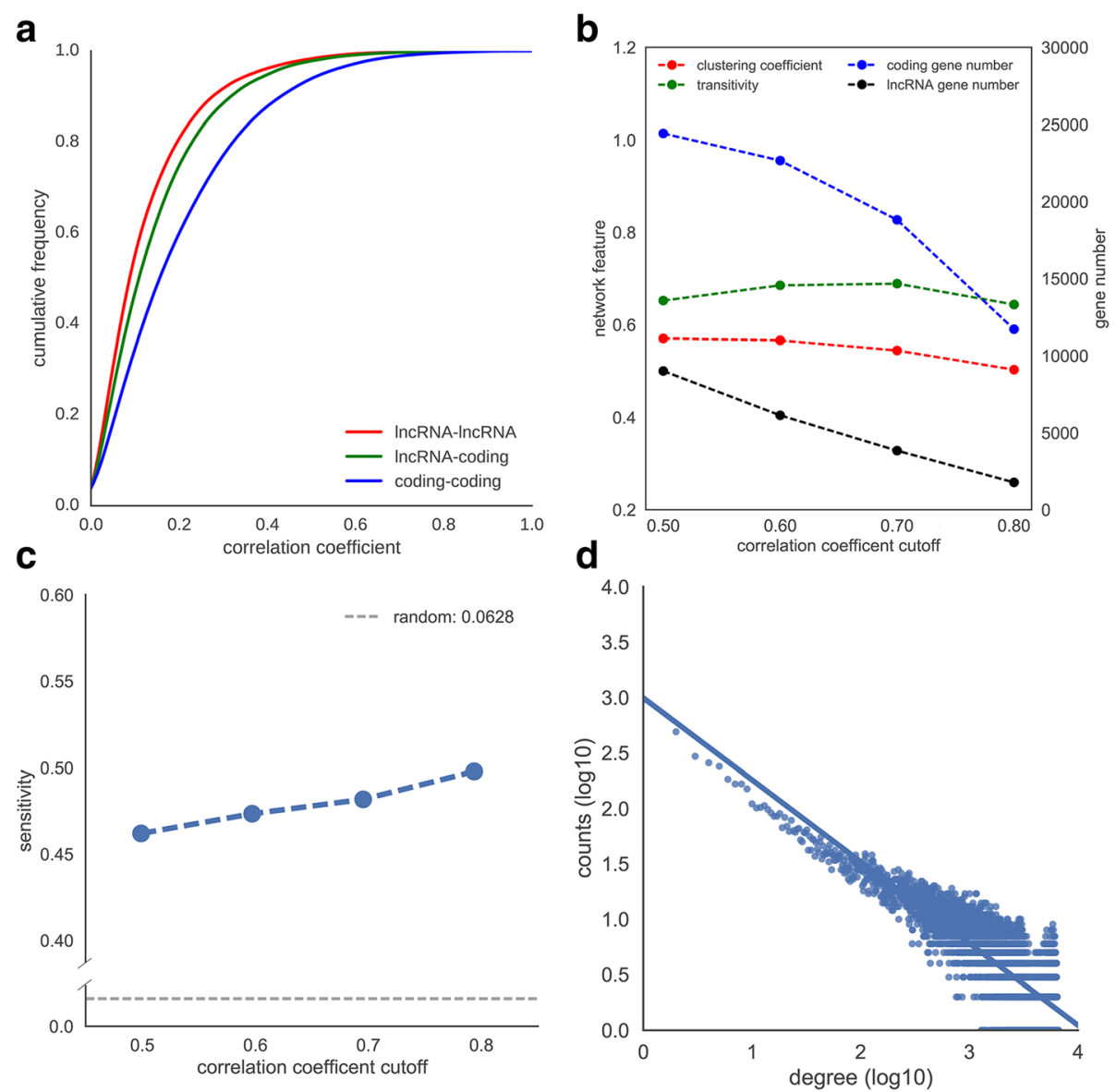

Fig. 3 Features of zebrafish coding-IncRNA gene co-expression network. a Cumulative distribution of gene expression Spearman's correlation coefficient. $\mathbf{b}$ Network statistics by different correlation coefficient cutoffs. $\mathbf{c}$ Evaluation of function prediction performance of the network with different cutoffs. $\mathbf{d}$ Network degree distribution (correlation coefficient cutoff $=0.5$ )

demonstrated that mammalian lncRNAs incline to be expressed specifically in the central nervous system and may play central roles in regulation of the development and function of nervous system $[29,30]$, we reasoned that zebrafish would be very compatible as a research platform for exploring lncRNA function in human nervous system.

\section{Conservation analysis of zebrafish IncRNAs}

For seeking out the putative mammalian orthologs of zebrafish lncRNAs, we used BLASTN to directly compare lncRNAs in zebrafish with that in human or mouse by turns. Those lncRNAs with best hits in bidirectional comparison were considered as lncRNA orthologs (E-value $<=10-5)$. In total, we identified 1828 conserved zebrafish lncRNA genes (1890 transcripts). Therein, 1258 zebrafish lncRNA transcripts have their corresponding orthologs in human; while 1099 zebrafish lncRNA transcripts have mouse orthologs; moreover, 467 zebrafish lncRNA transcripts have the overlapped orthologs in both human and mouse (Additional file 5).
When compared with all lncRNAs, the top ten GO BP terms for conserved zebrafish IncRNA are analogously associated with cell-signaling-related and chromosome-related processes, such as 'cell-cell signaling' $(P$-value $=1.49 \mathrm{e}-10)$ and 'transposition' ( $P$-value $=1.84 \mathrm{e}-05)$. There are a few of new GO BP terms added, such as 'macromolecular complex subunit organization' $(P$-value $=2.73 \mathrm{e}-10)$ and 'cellular component assembly' ( $P$-value $=3.14 \mathrm{e}-10)($ Fig. $4 \mathrm{c})$. Correspondingly, in the top ten KEGG pathways, several novel roles, like 'Spliceosome' ( $P$-value $=7.91 \mathrm{e}-29)$, 'RNA transport' $(P$-value $=6.05 \mathrm{e}-19)$ and 'Ribosome biogenesis in eukaryotes' $(P$-value $=2.7 \mathrm{e}-18)$, are present $($ Fig. $4 \mathrm{~d})$. This suggested that the conserved zebrafish lncRNAs are likely implicated in some of key biological processes in nucleus.

We also evaluated the full-transcript conservation levels of those conserved zebrafish lncRNAs using 8-way PhastCons scores (Fig. 5a). It was found that the PhastCons scores of conserved zebrafish lncRNA are close to that of zebrafish coding-gene, both of which are significantly greater than the entirety of all zebrafish lncRNAs (Kolmogorov-Smirnov test, $P$-value $=6.35 \mathrm{e}-155)$. Then, we examined the tissue 

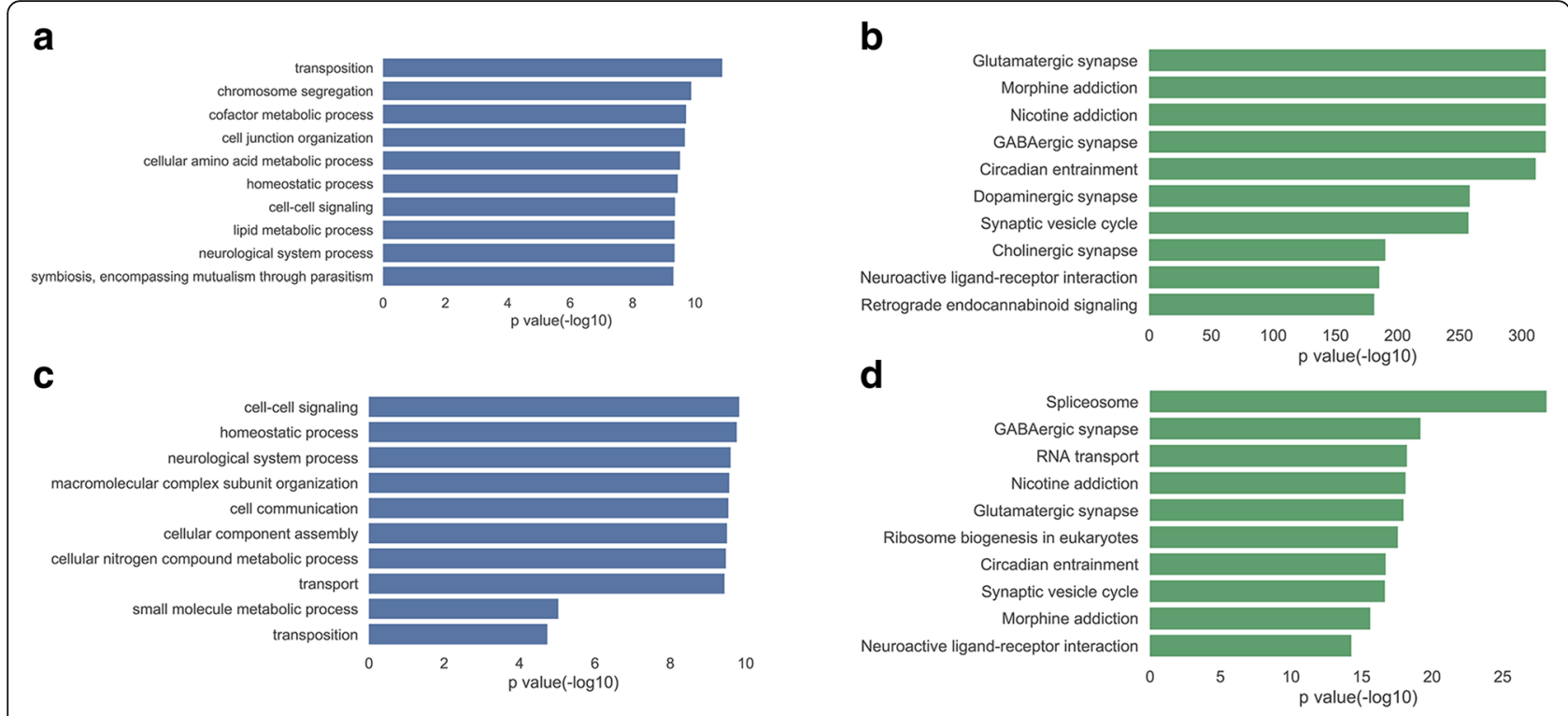

Fig. 4 Functional annotation of zebrafish IncRNAs. a LncRNA GO BP enrichment slim (top 10). b LncRNA KEGG pathway enrichment (top10). c Conserved IncRNA GO BP enrichment slim (top 10). d Conserved IncRNA KEGG pathway enrichment (top10)

expression specificity against diverse tissues and different conditions, amongst zebrafish lncRNA, conserved lncRNA and coding-gene. Unlike the unconserved ones, conserved lncRNAs are more resemblance of mRNA as displaying a less tissue-specific expression pattern (Kolmogorov-Smirnov test, $P$-value $=1.58 \mathrm{e}-12$ ), thereby may express and execute functions in more extensive tissues (Fig. 5b).

Furthermore, we investigated the multi-tissue expression profiles of conserved zebrafish lncRNAs, as compared with their mammalian counterparts (Fig. 5c). The conserved zebrafish lncRNAs, along with their mammalian orthologs, have a more strengthened correlation in tissue-expression profiles at heart, liver, muscle, brain and blood tissues, in comparison of random lncRNA-pairs between zebrafish and human (Kolmogorov-Smirnov test, $P$-value $=1.44 \mathrm{e}-07$ ). We have also evaluated the number of upstream transcription factor (TF) families shared by both conserved zebrafish lncRNA and its human counterpart (Fig. 5d). It was found that the conserved lncRNAs have a significant conformity with their mammalian counterparts in upstream regulatory pattern (Kolmogorov-Smirnov test, $P$-value $=1.95 \mathrm{e}-3$ ). Our results indicated that, as compared with their mammalian counterparts, the conservative property of conserved zebrafish lncRNAs reflects not only on gene sequence, probably also on the aspects of tissue expression and transcription regulation pattern.

\section{ZFLNCG05544 is a candidate IncRNA gene related to human neuron diseases}

Based on our own results on GO and KEGG annotation, multi-tissue expression profile and coding-lncRNA gene co-expression network, we made an attempt to explore the potential function of zebrafish lncRNA. Previous studies showed that a considerable amount of mammalian lncRNAs are specifically expressed in central nervous system, and thus may play important roles in nervous system development and maintenance [29, 30]. Likewise, we discovered that there are 2423 zebrafish lncRNAs related to neuron, according to their GO and/or KEGG annotation. After confining the tissue type and the expression level of zebrafish lncRNAs (Brain, average FPKM $>=10$ ), this number narrows down to 467 . Further, we examined the co-expression state between the latter and known human-neuron-disease-related coding-genes in zebrafish (data collected from ZFIN, http://zfin.org) [13]. Consequently, one lncRNA gene, ZFLNCG05544, attracted our attention.

ZFLNCG05544 locates in chromosome 9, containing two transcripts ZFLNCT08573 and ZFLNCT08574 with the length of $288 \mathrm{nt}$ and $402 \mathrm{nt}$ respectively (Fig. 6a). ZFLNCG05544 is highly expressed in Brain (average FPKM $=27.11$, look the details in Additional file 3). The top ten GO annotations of ZFLNCG05544 tend to be enriched in neuron-related processes, such as 'neuropeptide signaling pathway' $(P$-value $=2.5 \mathrm{e}-11)$, 'neurotransmitter transport' $(P$-value $=2.61 \mathrm{e}-11)$, 'modulation of synaptic transmission' $(P$-value $=3.59 \mathrm{e}-11)$, and 'synaptic signaling' ( $P$-value $=5.12 \mathrm{e}-11)$ (Fig. 6c). Equally, the top ten KEGG annotations of ZFLNCG05544 are also linked to neuron, such as 'Neuroactive ligand-receptor interaction' $(P$-value $=7.3 \mathrm{e}-42)$, 'Nicotine addiction' $(P$-value $=4.9 \mathrm{e}-31)$, 'Glutamatergic synapse' $(P$-value $=2.0 \mathrm{e}-30)$, 'Circadian 
a

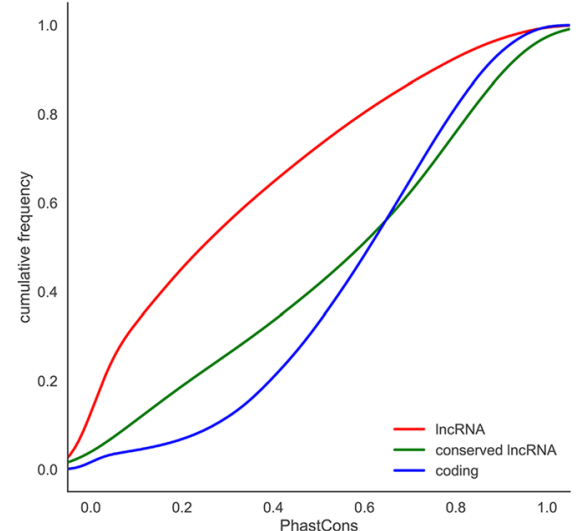

C

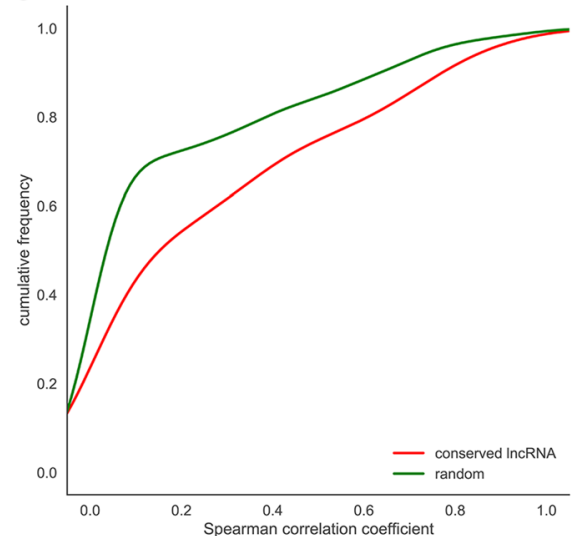

b

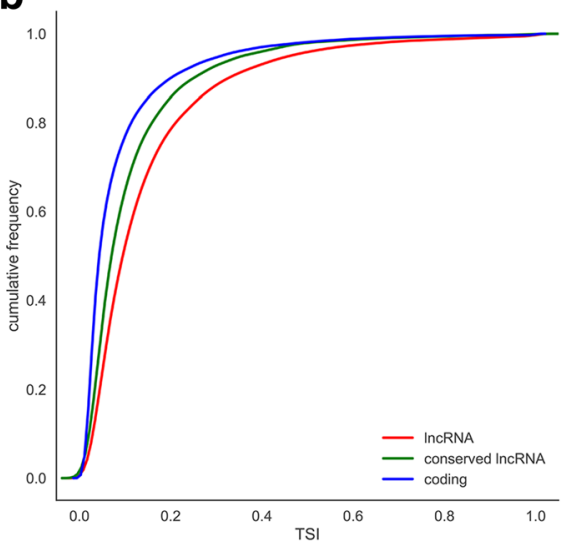

d

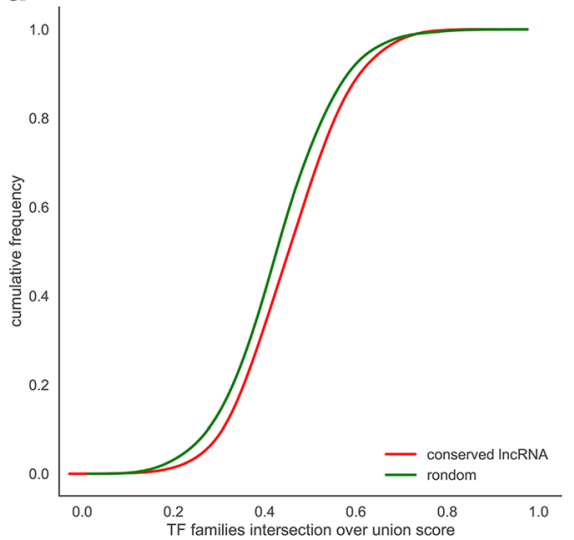

Fig. 5 Conservation analysis of zebrafish IncRNAs. a Cumulative distribution of conservation levels computed using PhastCons applied to the 8-way whole-genome. b Cumulative distribution of TSI (tissue specificity index). c Cumulative distribution of Spearman's correlation coefficient of gene expression. d Cumulative distribution of TF families' intersection over union score

entrainment' ( $P$-value $=7.4 \mathrm{e}-23)$, and 'Retrograde endocannabinoid signaling' $(P$-value $=2.5 \mathrm{e}-19)$ (Fig. 6d).

As shown by the co-expression sub-network centered around ZFLNCG05544, there is a concurrent expression of ZFLNCG05544 with many neuron-related codinggenes, especially those relevant to known human neuron diseases, like ace, appa, appb, ctsf, dnajc5aa, dnajc5ab, kif1b and tfg (Fig. 6b). For example, the amyloid precursor protein (APP) has been a focus of intense investigation because of its association with Alzheimer's disease [31]. Previous studies have shown that the zebrafish APP homologue, appb, is required for motor neuron guidance and synapse formation, and is essential for the formation of Mauthner cell in the hindbrain during development $[32,33]$. Another example is kinesin family member $1 \mathrm{~B}$ (kif1b), which has been associated with susceptibility to multiple sclerosis. Lyons et al. have found that kif1b is essential for mRNA localization in oligodendrocytes and development of myelinated axons in zebrafish [34]. ZFLNCG05544 is intensely linked to these neruronrelated coding-genes, as indicated by our coding-lncRNA gene co-expression network, implying its important roles in nervous system development and maintenance.

A very recent study gave a powerful support to our prediction of ZFLNCG05544 functions. Sarangdhar et al. demonstrated that zebrafish lncRNA durga modulates dendrite density through regulation of expression of kalrna, which is a coding-gene playing a key role in axonal development, nerve growth and synaptic remodeling [35]. Therein, lncRNA durga locates in the first exon of kalrna in an antisense orientation, and belongs to the catalogue of antisense lncRNA. By direct genomic mapping and BLAST alignment, we found that ZFLNCG05544 has the same location and strand direction in genome, when compared with lncRNA durga, but with a slight discrepancy in transcript length. Thus, they are probably different transcripts for the same gene. (Fig.6a). In view of the kalrna's association with many pathological conditions like schizophrenia and autism-spectrum disorders [36, 37], we thought that ZFLNCG05544 could be a proper candidate lncRNA gene related to multifarious human neuron diseases. 


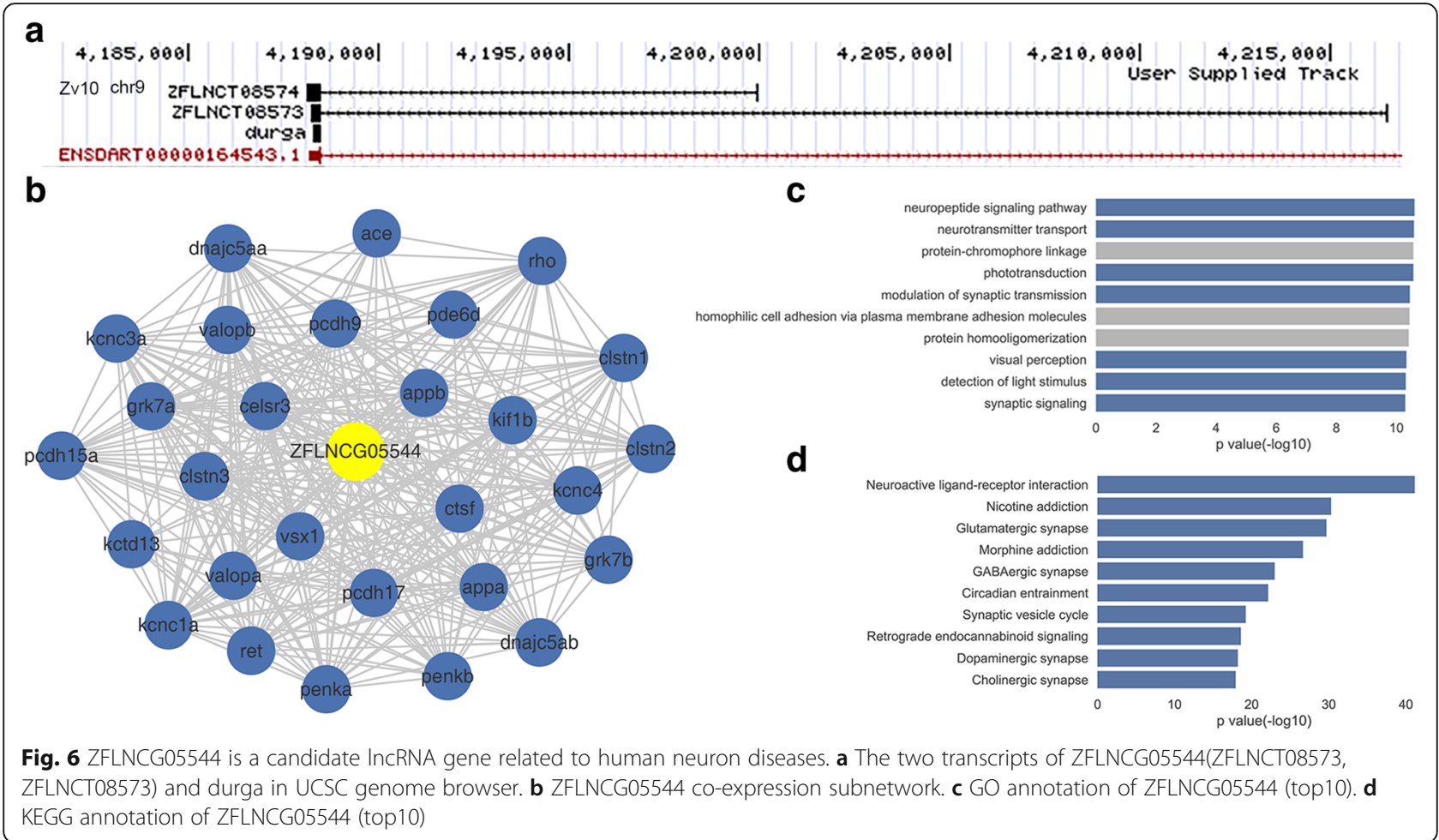

\section{ZFLNCG08251 is a human MALAT1 homolog in zebrafish}

Herein, we seek out 1890 conserved zebrafish lncRNAs, which have the corresponding mammalian orthologs. By further comparing them with those known functional human lncRNAs (101 entries in total) collected in lncRNAdb [38], twelve of conserved zebrafish lncRNAs in our own analysis hit their well-characterized human orthologs, including 7SK, 7SL, BC200, CCAT1, JPX, KRASP1, MALAT1, MASCRNA, MEGAMIND, OTX2OS1, PTENP1, RAB4BEGLN2. Amongst them, MALAT1 can parallel a lncRNA ZFLNCG08251 in zebrafish chromosome 14, a transcript of $7540 \mathrm{nt}$ length that is proximal to MALAT1 (8545 nt) (Additional file 2). This result is consistent with the discovery of Ulitsky et.al [20], in that a lncRNA transcript, in the same place of ZFLNCG08251, had been annotated to relevance of human and mouse's MATLAT1, yet fail to further function identification.

MALAT1 is a large, infrequently spliced non-coding RNA, which was closely related to various pathological processes, such as cancer, diabetes complications, and innate immunity [39-42]. Excitingly, the top ten GO annotations of ZFLNCG08251 are enriched in the exactly same categories of cancer, metabolism and immune (Fig. 7b), such as 'regulation of cell proliferation' $(P$-value $=1.7 \mathrm{e}-04)$, 'regulation of insulin-like growth factor receptor signaling pathway' (P-value $=3.8 \mathrm{e}-04)$, and 'immune system process' $(\mathrm{P}$-value $=5.6 \mathrm{e}-05)$. Moreover, its corresponding top ten KEGG pathways are also encapsulated within cancer, metabolism, immune annotation (Fig. 7c), such as 'Central carbon metabolism in cancer' $(P$-value $=8.7 \mathrm{e}-04)$, 'Insulin resistance' $(P$-value $=4.6 \mathrm{e}-03)$, and 'Intestinal immune network for IgA production' ( $P$-value $=6.4 \mathrm{e}-04)$.

In addition, the co-expression sub-network of ZFLNCG08251 shows that it is likely to actively involve in the pathological processes of cancer, diabetes complications, and innate immunity etc., as its human counterpart (Fig. 7a). Amongst the neighbor genes co-expressed with ZFLNCG08251, we detected extensively three types of genes, which are correlated to cancerous signaling pathway (e.g. fas, fes, egfra, map3k5, junba, tgfb1a, tnfsf12, tnfrsf1a, tnfrsf18, jak3, inhbaa), immunity (e.g. il15, irf1b, cd40, tbk1, mavs, socs2, socs3a, stat1a, nod2), and glycometabolism (e.g. insulin-like growth factorbinding protein - igfbp3, igfbp5a, igfbp6b, insulin-like growth factor - igf1, insulin receptor substrate - irs2). All these results hint that ZFLNCG08251, along with its human homolog MALAT1, not only has the sequence conservation, but also the conservative function.

\section{Discussion}

Zebrafish is recognized as one of the most important vertebrate model organisms, based on the fact that the development and function of zebrafish organs are strikingly similar to human. Combined with the ease of creating mutant or transgenic individual, zebrafish has been served as an important model system for studying human-disease-related protein-coding genes [14-16]. Nevertheless, the comparable study on zebrafish lncRNAs is still scanty, resulting in 


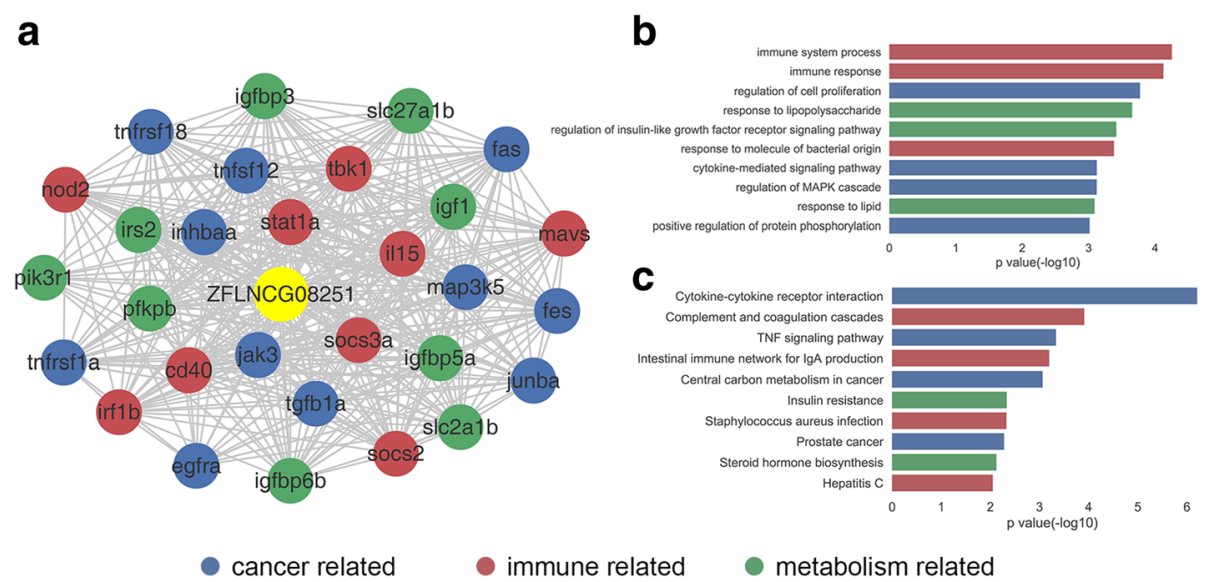

Fig. 7 ZFLNCG08251 is a human MALAT1 homolog in zebrafish. a ZFLNCG08251 co-expression subnetwork. b. GO annotation of ZFLNCG08251 (top10). c KEGG annotation of ZFLNCG08251 (top10)

part from the deficiency of systematic collection and characterization of IncRNAs, as well as the incomplete quantification of their expression profiles. On the other hand, the lack of systematic annotations of zebrafish lncRNAs' function and conservation also made a big obstacle to the follow-up study.

In this work, we provided by far the most comprehensive dataset of zebrafish lncRNAs, which is consist of multiple information of zebrafish lncRNAs, like expression profile, co-expression network, function and conservation annotation etc. Our functional analysis uncovered that, similar to mammalian IncRNA, zebrafish lncRNAs also tend to play roles in regulation of the development and function of nervous system; additionally, conservation analysis indicated that the conserved zebrafish IncRNAs have both sequential and functional conservative with their mammalian counterparts. Therefore, we inferred that zebrafish would be an applicable platform for exploring lncRNA function in development processes and human diseases.

Unlike the protein-coding gene, it is very difficult to directly annotate lncRNA's functions by sequence analysis, due to the lack of information about lncRNA functional and/or structural domain. The existing studies had revealed that mammalian lncRNAs often execute functions with their protein partners, this fact is verified by our analysis of coding-lncRNA gene co-expression in zebrafish. On account of this, the annotation for lncRNA often utilizes the relevant co-expressed coding-genes in network as to enrich lncRNA's function [43, 44]. Compared with human and mouse, the high-throughput sequencing data of zebrafish lncRNA transcriptome are still in less scale. We believed that, in the future, accumulating data of zebrafish transcriptome would further strength our power of lncRNA function prediction based on co-expression network.

Zebrafish shares a high genetic similarity to human, and lots of known human-disease-related coding-genes have functional homologs in zebrafish. However, the conservative of zebrafish lncRNAs is worthy to further gain insight [45]. Through direct BLASTN search, we found 1890 conserved zebrafish lncRNAs, accounting for about $8.9 \%$ of total zebrafish lncRNAs $(1890 / 21,128)$, this result is consistent with the discovery on conservation analysis by Ulitsky et.al $(5.3 \%, 29 / 550)$ [20]. In view of the low conservation of lncRNA sequence, syntenic conservation analysis will facilitate to search out the homologs of less conserved lncRNAs, other than the traditional direct BLASTN search [46]. Besides the increased sensitivity of conservation analysis, how to ensure the analysis' specificity is still an open question.

\section{Conclusions}

In summary, by integrative data analysis and construction of coding-lncRNA gene co-expression network, we gained by far the most comprehensive dataset of zebrafish lncRNAs, as well as their systematic annotations and comprehensive analyses on function and conservation. Our study provides some important insights into the functional roles of zebrafish lncRNAs and their possible application for studying human diseases. We anticipate that this study will provide a reliable zebrafish-based platform to deeply explore lncRNA function and mechanism, and make a roadmap to investigate the commonality of lncRNAs' function and conservation between zebrafish and human.

\section{Methods}

Data sources

RNA-Seq data are downloaded from NCBI SRA database; known zebrafish lncRNAs are collected from NCBI [24], Ensembl [25], NONCODEv4 [22], zflncRNApedia [23] and literatures [20,21] (See Additional file 1 for details). 


\section{RNA-seq data analysis pipeline}

SRA format files were dumped to FASTQ format files by SRA-Toolkit. Low quality reads were trimmed by Trimmomatic (Version 0.32) [47]. RNA-Seq reads were mapped to zebrafish genome (Zv9) using Tophat2 (Version 2.0.13) [48], then transcriptome was assembled by Cufflinks (Version 2.2.1) [49]. Multiple-exon transcripts were considered as being expressed if they had an FPKM greater than 0.1. For single-exon transcript, more rigorously, FPKM should be greater than 5 and transcript length greater than 2000. Those foregone coding-genes or transcripts with size less than 200 nt were filtered out. Then, IncRNA candidates were identified by CPC (Version 0.9-r2) [50] and CNCI (Version 2) [51]. At last, all zebrafish lncRNAs stemming from RNA-seq datasets and other publicly available sources were integrated together using the Cuffmerge program in the Cufflinks suite.

\section{Coding-IncRNA gene co-expression network construction} The expression profile of zebrafish lncRNAs and codinggenes were quantified by Cuffnorm program in the Cufflinks suite and then scaled by upper-quartile normalization (Eq. 1). We then calculated the Spearman's correlation coefficient and its corresponding $P$-value (Eq. 2) between the expression profiles of each gene-pair using the in-house Perl script. Only gene-pair with an adjusted P-value of 0.01 or less and with a Spearman's correlation coefficient no less than 0.5 is regarded as co-expression in our coding-lncRNA gene co-expression network.

$$
\rho_{i}=\frac{\frac{1}{n} \sum_{i}^{n} k_{i}}{k_{i}}
$$

For each sample i, $k_{i}$ is its upper quartile of all gene expression values different from $0, \mathrm{n}$ is the number of all samples, $\rho_{i}$ is the scaling factor for sample i. The upper quartile normalization for all gene expression values in sample $\mathrm{i}$ is obtained by multiplying them to the scaling factor $\rho_{i}$.

$$
\left\{\begin{array}{l}
\mathrm{Rs}=\frac{\sum_{i}\left(x_{i}-\bar{x}\right)\left(y_{i}-\bar{y}\right)}{\sqrt{\sum_{i}\left(x_{i}-\bar{x}\right)^{2} \sum_{i}\left(y_{i}-\bar{y}\right)^{2}}} \\
\mathrm{~F}(\mathrm{Rs})=\frac{1}{2} \ln \frac{1+\mathrm{Rs}}{1-\mathrm{Rs}} \\
\mathrm{Z}=\sqrt{\frac{\mathrm{n}-3}{1.06} \mathrm{~F}(\mathrm{Rs})}
\end{array}\right.
$$

Where $\mathrm{x}$ or $\mathrm{y}$ represents the vector of the ranked expression value of each gene, Rs is the Spearman's correlation coefficient between $\mathrm{x}$ and $\mathrm{y}, x_{i}$ or $y_{i}$ stands for the rank of each expression value, $\bar{x}$ or $\bar{y}$, is the mean value of these ranks. $F$ (Rs) is the Fisher transformation of Rs, and $\mathrm{n}$ is the sample size i.e. the vector length. The corresponding $P$-value of each $R$ s is calculated from $\mathrm{Z}$, which is a $\mathrm{Z}$-score for Rs that approximately follows a standard normal distribution under the null hypothesis of statistical independence [52, 53].

\section{Network statistics}

The clustering coefficient score $\mathrm{C}$ for a graph is the average of local clustering coefficient values $C_{i}$ of each node (Eq. 3). The transitivity $\mathrm{T}$ of a graph is based on the relative number of triangles in the graph, compared to total number of connected triples of nodes (Eq. 4).

$$
\left\{\begin{array}{c}
\mathrm{C}_{\mathrm{i}}=\frac{\text { number of triangles connected to node } i}{\text { number of triples centered around node } i} \\
C=\frac{1}{\mathrm{n}} \sum_{\mathrm{i}=1}^{\mathrm{n}} \mathrm{C}_{\mathrm{i}}
\end{array}\right.
$$

Where If node $\mathrm{i}$ has two neighbor nodes and these two nodes are also connected, there is a triangle connected to node $i$, a triple centered around node $i$ is a set of two edges connected to node $\mathrm{i}, \mathrm{n}$ is the number of node in the network. By definition, $0 \leq C_{i} \leq 1$ and $0 \leq \mathrm{C} \leq 1$.

$$
T=\frac{3 \times \text { number of triangles in the network }}{\text { number of connected triples of nodes in the network }}
$$

The factor of " 3 " in number accounts for the fact that each triangle contributes to three different connected triples in the graph, one centered at each node of the triangle. With this definition, $0 \leq T \leq 1$, and $T=1$ if the network contains all possible edges.

\section{LncRNA functional annotation}

The GO annotation of zebrafish coding-gene was downloaded from Gene Ontology Consortium (only biological process annotations were considered). While, GO annotation of zebrafish lncRNA was predicted using the goatools (version 0.6.4) [54], which determines the GO annotation of one gene in our network according to the GO annotations of its immediate neighbor genes $(P$-value $<0.05)$.

The KEGG annotation of zebrafish coding-gene was obtained from KEGG Automatic Annotation Server using zebrafish coding-gene sequence [55]. While, KEGG annotation of zebrafish lncRNA was predicted using the in-house Python script. The KEGG annotation of one gene in our network was determined by the enrichment of KEGG annotations according to its immediate neighborhood ( $p$-value $<0.05)$, when using hypergeometric distribution (Eq. 5). 


$$
\mathrm{P}=1-\sum_{i=0}^{k-1} \frac{\left(\frac{M}{i}\right)\left(\frac{N-M}{n-i}\right)}{\left(\frac{N}{n}\right)}
$$

In this equation, $\mathrm{N}$ is the total number of genes in the network, $\mathrm{M}$ is the total number of genes having one certain KEGG annotation, $\mathrm{n}$ is the number of a gene's immediate neighbors and $\mathrm{k}$ is the number of neighbor genes having one certain KEGG annotation.

\section{Analysis of conserved IncRNAs}

Zebrafish lncRNAs' putative mammalian orthologs were inferred from bidirectional best hits in direct comparison of zebrafish lncRNA and human lncRNA or mouse lncRNA with BLASTN using a relatively non-stringent E-value threshold $10^{-5}$. Zebrafish lncRNAs' conservation levels were evaluated using 8-way PhastCons score [56].

Using expression profiles of all SRA runs, the tissue specificity of zebrafish lncRNAs' gene expression was calculated by TSI (Eq. 6).

$$
\mathrm{TSI}=\frac{\max \left(\mathrm{x}_{\mathrm{i}}\right)}{\sum_{\mathrm{i}} \mathrm{x}_{\mathrm{i}}}
$$

Where TSI is the tissue specificity index, $x_{\mathrm{i}}$ is the expression value.

To obtain the correlation of tissue expression among conserved lncRNAs, Spearman's correlation coefficient scores were calculated for each conserved zebrafish lncRNA and its human counterpart. The tissue-expression profile data of zebrafish come from five organs, including heart, liver, muscle, brain and blood, as reported by Kaushik et.al. [57]; while, that of human from Human body map.

To estimate the conformity of upstream (from -500 to +100 ) regulatory patterns of conserved lncRNAs, we evaluated the number of upstream transcription factor (TF) families shared by both conserved zebrafish lncRNA and its human counterpart using intersection over union score (Eq. 7). TF-binding site prediction was performed using the HOMER tool [58].

$$
\mathrm{S}=\frac{\cap\left(\mathrm{TF}_{\mathrm{Z}}, \mathrm{TF}_{\mathrm{H}}\right)}{\mathrm{u}\left(\mathrm{TF}_{\mathrm{Z}}, \mathrm{TF}_{\mathrm{H}}\right)}
$$

Where $S$ is the intersection over union score of upstream TF families shared by both conserved zebrafish lncRNA $\left(\mathrm{TF}_{\mathrm{Z}}\right)$ and its human counterpart $\left(\mathrm{TF}_{\mathrm{H}}\right)$.

\section{Additional files}

Additional file 1: Data source. (XLSX $33 \mathrm{~kb})$

Additional file 2: IncRNA dataset. (ZIP 10705 kb)
Additional file 3: IncRNA-coding expression profiles. Replicate samples are merged. (ZIP 13305 kb)

Additional file 4: IncRNA GO and KEGG annotation. (ZIP 17127 kb)

Additional file 5: Conserved IncRNA. (XLSX 84 kb)

Abbreviations

BP: Biological Process; FPKM: Fragments Per Kilobase Million; GO: Gene Ontology; KEGG: Kyoto Encyclopedia of Genes and Genomes; LncRNA: Long non-coding RNA; TF: Transcription Factor; TSI: Tissue Specificity Index

\section{Acknowledgements}

RNA-Seq data analysis supported by HPC Platform, The Public Technology Service Center of Xishuangbanna Tropical Botanical Garden (XTBG), CAS, China.

\section{Funding}

This work was supported by the National Natural Science Foundation of China (No. 31471220, 91440113), Start-up Fund from Xishuangbanna Tropical Botanical Garden, 'Top Talents Program in Science and Technology' from Yunnan Province, the Opening Fund of State Key Laboratory of Developmental Biology of Freshwater Fish (2017KF003), the Scientific Research Fund of Hunan Provincial Education Department (14B114), and the Cooperative Innovation Center of Engineering and New Products for Developmental Biology of Hunan Province (20134486). Publication of this article was sponsored by the National Natural Science Foundation of China (No. 91440113).

\section{Availability of data and materials}

All data generated or analyzed during this study are included in this published article.

\section{About this supplement}

This article has been published as part of BMC Genomics Volume 19 Supplement 2, 2018: Selected articles from the 16th Asia Pacific Bioinformatics Conference (APBC 2018): genomics. The full contents of the supplement are available online at https://bmcgenomics.biomedcentral.com/ articles/supplements/volume-19-supplement-2

\section{Authors' contributions}

$\mathrm{CL}$ and $\mathrm{XH}$ conceived, designed, and supervised this study. WC, XZ and JL performed the most data analysis and contributed equally to this work. SH and SX participate in the discussion and provide valuable advice and practical contributions. $W C$, $J$ and $C L$ wrote the first draft of the article, all authors reviewed, edited and approved the manuscript.

Ethics approval and consent to participate

No animals or other organisms were used in this study.

Consent for publication

Not applicable.

Competing interests

The authors declare that they have no competing interests.

\section{Publisher's Note}

Springer Nature remains neutral with regard to jurisdictional claims in published maps and institutional affiliations.

Published: 9 May 2018

\section{References}

1. Wang KC, Chang HY. Molecular mechanisms of long noncoding RNAs. Mol Cell. 2011;43(6):904-14

2. Iyer MK, Niknafs YS, Malik R, Singhal U, Sahu A, Hosono Y, Barrette TR, Prensner JR, Evans JR, Zhao S, et al. The landscape of long noncoding RNAs in the human transcriptome. Nat Genet. 2015:47(3):199-208.

3. Ulitsky I, Bartel DP. lincRNAs: genomics, evolution, and mechanisms. Cell. 2013;154(1):26-46.

4. Bu D, Yu K, Sun S, Xie C, Skogerbo G, Miao R, Xiao H, Liao Q, Luo H, Zhao G, et al. NONCODE v3.0: integrative annotation of long noncoding RNAs. Nucleic Acids Res. 2012;40(Database issue):D210-5. 
5. Zhao Y, Li H, Fang S, Kang Y, Wu W, Hao Y, Li Z, Bu D, Sun N, Zhang MQ, et al. NONCODE 2016: an informative and valuable data source of long noncoding RNAs. Nucleic Acids Res. 2016;44(D1):D203-8.

6. Liu C, Bai B, Skogerbo G, Cai L, Deng W, Zhang Y, Bu D, Zhao Y, Chen R. NONCODE: an integrated knowledge database of non-coding RNAs. Nucleic Acids Res. 2005;33(Database issue):D112-5.

7. Guttman M, Donaghey J, Carey BW, Garber M, Grenier JK, Munson G, Young $G$, Lucas $A B$, Ach $R$, Bruhn $L$, et al. lincRNAs act in the circuitry controlling pluripotency and differentiation. Nature. 2011;477(7364):295-300.

8. Cabili MN, Trapnell C, Goff L, Koziol M, Tazon-Vega B, Regev A, Rinn JL. Integrative annotation of human large intergenic noncoding RNAs reveals global properties and specific subclasses. Genes Dev. 2011;25(18):1915-27.

9. Gupta RA, Shah N, Wang KC, Kim J, Horlings HM, Wong DJ, Tsai MC, Hung T, Argani $P$, Rinn $J$, et al. Long non-coding RNA HOTAIR reprograms chromatin state to promote cancer metastasis. Nature. 2010;464(7291):1071-6.

10. Li J, Xuan Z, Liu C. Long non-coding RNAs and complex human diseases. Int J Mol Sci. 2013;14(9):18790-808.

11. Wapinski O, Chang HY. Long noncoding RNAs and human disease. Trends Cell Biol. 2011;21(6):354-61.

12. Bhan A, Mandal SS. Long noncoding RNAs: emerging stars in gene regulation, epigenetics and human disease. ChemMedChem. 2014;9(9): 1932-56.

13. Bradford Y, Conlin T, Dunn N, Fashena D, Frazer K, Howe DG, Knight J, Mani P, Martin R, Moxon SA, et al. ZFIN: enhancements and updates to the Zebrafish model organism database. Nucleic Acids Res. 2011;39(Database issue):D822-9.

14. Zon LI. Zebrafish: a new model for human disease. Genome Res. 1999;9(2):99-100.

15. Dooley K, Zon LI. Zebrafish: a model system for the study of human disease. Curr Opin Genet Dev. 2000;10(3):252-6.

16. Lieschke GJ, Currie PD. Animal models of human disease: zebrafish swim into view. Nat Rev Genet. 2007;8(5):353-67.

17. Chiabrando D, Mercurio S, Tolosano E. Heme and erythropoieis: more than a structural role. Haematologica. 2014;99(6):973-83.

18. Bournele D, Beis D. Zebrafish models of cardiovascular disease. Heart Fail Rev. 2016;21(6):803-13.

19. Cirio MC, de Caestecker MP, Hukriede NA. Zebrafish models of kidney damage and repair. Curr Pathobiol Rep. 2015;3(2):163-70.

20. Ulitsky I, Shkumatava A, Jan CH, Sive H, Bartel DP. Conserved function of lincRNAs in vertebrate embryonic development despite rapid sequence evolution. Cell. 2011;147(7):1537-50.

21. Pauli A, Valen E, Lin MF, Garber M, Vastenhouw NL, Levin JZ, Fan L, Sandelin A, Rinn JL, Regev A, et al. Systematic identification of long noncoding RNAs expressed during zebrafish embryogenesis. Genome Res. 2012;22(3):577-91.

22. Xie C, Yuan J, Li H, Li M, Zhao G, Bu D, Zhu W, Wu W, Chen R, Zhao Y. NONCODEv4: exploring the world of long non-coding RNA genes. Nucleic Acids Res. 2014;42(Database issue):D98-103.

23. Dhiman H, Kapoor S, Sivadas A, Sivasubbu S, Scaria V. zflncRNApedia: a comprehensive online resource for Zebrafish long non-coding RNAs. PLoS One. 2015;10(6):e0129997.

24. Pruitt KD, Tatusova T, Brown GR, Maglott DR. NCBI reference sequences (RefSeq): current status, new features and genome annotation policy. Nucleic Acids Res. 2012;40(Database issue):D130-5.

25. Yates A, Akanni W, Amode MR, Barrell D, Billis K, Carvalho-Silva D, Cummins C, Clapham P, Fitzgerald S, Gil L, et al. Ensembl 2016. Nucleic Acids Res. 2016:44(D1):D710-6.

26. Plath K, Mlynarczyk-Evans S, Nusinow DA, Panning B. Xist RNA and the mechanism of $X$ chromosome inactivation. Annu Rev Genet. 2002; 36:233-78.

27. Sleutels F, Zwart R, Barlow DP. The non-coding air RNA is required for silencing autosomal imprinted genes. Nature. 2002;415(6873):810-3.

28. Lee S, Kopp F, Chang TC, Sataluri A, Chen B, Sivakumar S, Yu H, Xie Y, Mendell JT. Noncoding RNA NORAD regulates genomic stability by sequestering PUMILIO proteins. Cell. 2016;164(1-2):69-80.

29. Mercer TR, Dinger ME, Sunkin SM, Mehler MF, Mattick JS. Specific expression of long noncoding RNAs in the mouse brain. Proc Natl Acad Sci U S A. 2008;105(2):716-21.

30. Chodroff RA, Goodstadt L, Sirey TM, Oliver PL, Davies KE, Green ED, Molnar Z, Ponting CP. Long noncoding RNA genes: conservation of sequence and brain expression among diverse amniotes. Genome Biol. 2010;11(7):R72.

31. O'Brien RJ, Wong PC. Amyloid precursor protein processing and Alzheimer's disease. Annu Rev Neurosci. 2011;34:185-204.
32. Abramsson A, Kettunen P, Banote RK, Lott E, Li M, Arner A, Zetterberg H. The zebrafish amyloid precursor protein-b is required for motor neuron guidance and synapse formation. Dev Biol. 2013;381(2):377-88.

33. Banote RK, Edling $M$, Eliassen $F$, Kettunen $P$, Zetterberg $H$, Abramsson $A$. Beta-Amyloid precursor protein-b is essential for Mauthner cell development in the zebrafish in a notch-dependent manner. Dev Biol. 2016; 413(1):26-38

34. Lyons DA, Naylor SG, Scholze A, Talbot WS. Kif1b is essential for mRNA localization in oligodendrocytes and development of myelinated axons. Nat Genet. 2009;41(7):854-8.

35. Sarangdhar MA, Chaubey D, Bhatt A, Km M, Kumar M, Ranjan S, Pillai B. A novel long non-coding RNA, durga modulates dendrite density and expression of kalirin in Zebrafish. Front Mol Neurosci. 2017;10:95.

36. Deo AJ, Cahill ME, Li S, Goldszer I, Henteleff R, Vanleeuwen JE, Rafalovich I, Gao R, Stachowski EK, Sampson AR, et al. Increased expression of Kalirin-9 in the auditory cortex of schizophrenia subjects: its role in dendritic pathology. Neurobiol Dis. 2012:45(2):796-803.

37. Grubisha MJ, Lin CW, Tseng GC, Penzes P, Sibille E, Sweet RA. Agedependent increase in Kalirin-9 and Kalirin-12 transcripts in human orbitofrontal cortex. Eur J Neurosci. 2016;44(7):2483-92.

38. Quek XC, Thomson DW, Maag JL, Bartonicek N, Signal B, Clark MB, Gloss BS, Dinger ME. IncRNAdb v2.0: expanding the reference database for functional long noncoding RNAs. Nucleic Acids Res. 2015;43(Database issue):D168-73.

39. Wu Y, Huang C, Meng X, Li J. Long noncoding RNA MALAT1: insights into its biogenesis and implications in human disease. Curr Pharm Des. 2015; 21(34):5017-28.

40. Gutschner T, Hammerle M, Eissmann M, Hsu J, Kim Y, Hung G, Revenko A, Arun G, Stentrup M, Gross M, et al. The noncoding RNA MALAT1 is a critical regulator of the metastasis phenotype of lung cancer cells. Cancer Res. 2013;73(3):1180-9.

41. Gast M, Schroen B, Voigt A, Haas J, Kuehl U, Lassner D, Skurk C, Escher F, Wang $X$, Kratzer A, et al. Long noncoding RNA MALAT1-derived mascRNA is involved in cardiovascular innate immunity. J Mol Cell Biol. 2016;8(2):178-81.

42. Liu JY, Yao J, Li XM, Song YC, Wang XQ, Li YJ, Yan B, Jiang Q. Pathogenic role of IncRNA-MALAT1 in endothelial cell dysfunction in diabetes mellitus. Cell Death Dis. 2014;5:e1506

43. Liao Q, Xiao H, Bu D, Xie C, Miao R, Luo H, Zhao G, Yu K, Zhao H, Skogerbo $\mathrm{G}$, et al. ncFANs: a web server for functional annotation of long non-coding RNAs. Nucleic Acids Res. 2011;39(Web Server issue):W118-24.

44. Liao Q, Liu C, Yuan X, Kang S, Miao R, Xiao H, Zhao G, Luo H, Bu D, Zhao $\mathrm{H}$, et al. Large-scale prediction of long non-coding RNA functions in a coding-non-coding gene co-expression network. Nucleic Acids Res. 2011:39(9):3864-78.

45. Hezroni H, Koppstein D, Schwartz MG, Avrutin A, Bartel DP, Ulitsky I. Principles of long noncoding RNA evolution derived from direct comparison of transcriptomes in 17 species. Cell Rep. 2015;11(7):1110-22.

46. Necsulea A, Soumillon M, Warnefors M, Liechti A, Daish T, Zeller U, Baker JC, Grutzner F, Kaessmann H. The evolution of IncRNA repertoires and expression patterns in tetrapods. Nature. 2014;505(7485):635-40.

47. Bolger AM, Lohse M, Usadel B. Trimmomatic: a flexible trimmer for Illumina sequence data. Bioinformatics. 2014;30(15):2114-20.

48. Kim D, Pertea G, Trapnell C, Pimentel H, Kelley R, Salzberg SL. TopHat2: accurate alignment of transcriptomes in the presence of insertions, deletions and gene fusions. Genome Biol. 2013;14(4):R36.

49. Trapnell C, Williams BA, Pertea G, Mortazavi A, Kwan G, van Baren MJ, Salzberg SL, Wold BJ, Pachter L. Transcript assembly and quantification by RNA-Seq reveals unannotated transcripts and isoform switching during cell differentiation. Nat Biotechnol. 2010;28(5):511-5.

50. Kong L, Zhang Y, Ye ZQ, Liu XQ, Zhao SQ, Wei L, Gao G. CPC: assess the protein-coding potential of transcripts using sequence features and support vector machine. Nucleic Acids Res. 2007;35(Web Server issue):W345-9.

51. Sun L, Luo H, Bu D, Zhao G, Yu K, Zhang C, Liu Y, Chen R, Zhao Y. Utilizing sequence intrinsic composition to classify protein-coding and long noncoding transcripts. Nucleic Acids Res. 2013:41(17):e166.

52. Choi SC. Tests of equality of dependent correlation coefficients. Biometrika. 1977;64(3):645-7.

53. Fieller EC, Hartley HO, Pearson ES. Tests for rank correlation coefficients. I Biometrika. 1957;44(3-4):470-81.

54. Haibao T, Debra K, Brent P, Patrick F, Kenta S, Fidel R, Jeff Y, Chris M. GOATOOLS: tools for gene ontology. 2015. https://doi.org/10.5281/zenodo.31628. 
55. Moriya Y, Itoh M, Okuda S, Yoshizawa AC, Kanehisa M. KAAS: an automatic genome annotation and pathway reconstruction server. Nucleic Acids Res. 2007;35(Web Server issue):W182-5.

56. Siepel A, Bejerano G, Pedersen JS, Hinrichs AS, Hou M, Rosenbloom K, Clawson H, Spieth J, Hillier LW, Richards S, et al. Evolutionarily conserved elements in vertebrate, insect, worm, and yeast genomes. Genome Res. 2005;15(8):1034-50.

57. Kaushik K, Leonard VE, Kv S, Lalwani MK, Jalali S, Patowary A, Joshi A, Scaria $\checkmark$, Sivasubbu S. Dynamic expression of long non-coding RNAs (IncRNAs) in adult zebrafish. PLoS One. 2013;8(12):e83616.

58. Heinz S, Benner C, Spann N, Bertolino E, Lin YC, Laslo P, Cheng JX, Murre C, Singh $H$, Glass CK. Simple combinations of lineage-determining transcription factors prime cis-regulatory elements required for macrophage and B cell identities. Mol Cell. 2010;38(4):576-89.

Submit your next manuscript to BioMed Central and we will help you at every step:

- We accept pre-submission inquiries

- Our selector tool helps you to find the most relevant journal

- We provide round the clock customer support

- Convenient online submission

- Thorough peer review

- Inclusion in PubMed and all major indexing services

- Maximum visibility for your research

Submit your manuscript at www.biomedcentral.com/submit
Biomed Central 